\title{
IGPC-QFT Cascade Control and Its Application in Superheated Steam Temperature Control System
}

\author{
Liu Xuyang1, a \\ ${ }^{1}$ Wuhan University of Science and Technology, Heping Avenue 947,Qingshan District,Wuhan \\ Hubei, China)
}

a393932763@qq.com,

\begin{abstract}
Keywords: superheated steam temperature control; quantitative feedback theory; improved implicit generalized predictive control algorithm; cascade control; suppressed overshoot.

Abstract. The superheated steam temperature control system is a complicated controlled object with large inertia and large time delay characteristics, and its model parameters change with the load. For this nonlinear system, an IGPC-QFT cascade control algorithm was proposed. The quantitative feedback theory (QFT) was used to suppress the uncertainties and disturbance in the leading region of the system. A simple method for QFT controller design was proposed to avoid the tedious shaping process of the QFT controller design. An improved implicit generalized predictive self-tuning controller (IGPC) was used to suppress the overshoot and overcome the large inertia and large time delay in the inert region. The numerical simulations show that the proposed IGPC-QFT algorithm can give the controlled system better dynamic performance than traditional GPC-P under different load and disturbance.
\end{abstract}

\section{Introduction}

The superheated steam temperature control system is a complicated object with large inertia and large time delay, and its model parameters change with the load. Under different load and disturbance, the PID control algorithm can't meet the performance requirements ${ }^{[1]}$. In order to solve this problem, a variety of control strategies have been proposed. In [2], the PID parameters are adjusted by the comprehensive satisfaction function determined by the New Luus-Jaakola (NLJ) algorithm to reduce the influence of changed model parameters. In [3], the Adaptive Predictive Expert (ADEX) control method is used to control the steam temperature to maintain the thermal pressure minimum. In [4], an improved generalized predictive control algorithm is proposed. Improved least squares method is used to estimate the model parameters and reduce the influence of time delay and time-varying. In the actual industrial control, the superheated steam temperature control system is sensitive to the generation of overshoot. However, when the traditional GPC algorithm is used in the superheated steam temperature control system, the output will appear varying degree of overshoot. The quantitative feedback theory (QFT) can quantify the feedback information in the frequency domain, and design a robust controller in the case of uncertainty of the model parameters ${ }^{[5]}$. It is widely used in various fields. In [6], it is used to design the automatic landing controller. In [7], it is used to design a robust controller for hydraulic robots. The process of controller design is tedious ${ }^{[8]}$. Therefore, a method is needed to avoid it.

Aiming at the characteristics of superheated steam temperature system and two kinds of algorithm, the IGPC-QFT cascade control algorithm is proposed in this paper.

\section{Quantitative Feedback Theory (QFT) and Controller Design Method}

Fundamentals of QFT. QFT is a technology based on the frequency domain theory, using the Nichols chart to design controller.

Structure of single-input and single-output QFT system is shown in Fig. $1^{[9]}$.

Where $\mathrm{P}$ represents object with unknown parameters or structure, $\mathrm{C}$ represents the controller, $\mathrm{F}$ represents the filter, $d_{1}$ and $d_{2}$ represent interference, $\mathrm{R}$ represents the reference and $\mathrm{Y}$ represents the output of system. 


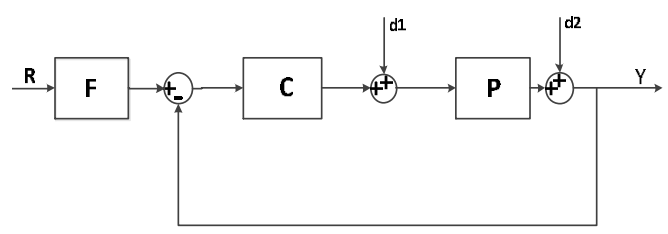

Fig. 1 Structure of single-input and single-output QFT system

Design Process of QFT. An object with uncertain parameters can be represented as:

$$
\vartheta=\left\{P(s)=\sum_{i} a_{i} s^{i} / \sum_{j} b_{j} s^{j}:\left[a_{i}, b_{j}\right] \in \Delta\right\} .
$$

$\left[a_{i}, b_{j}\right] \in \Delta$ indicates that the region of the parameters.

Common closed-loop performance indexes are:

1) Robust stability margin index

$$
\forall P(j \omega) \in \vartheta,\left|\frac{C(j \omega) P(j \omega)}{1+C(j \omega) P(j \omega)}\right| \leq M_{0} \cdot
$$

Where $M_{0}$ represents the resonant amplitude of the system.

2) Input interference rejection index

$$
\forall P(j \omega) \in \vartheta,\left|\frac{P(j \omega)}{1+C(j \omega) P(j \omega)}\right| \leq W_{1} .
$$

Where $W_{1}$ represents the input interference rejection coefficient.

3) Output interference rejection index

$$
\forall P(j \omega) \in \vartheta,\left|\frac{1}{1+C(j \omega) P(j \omega)}\right| \leq W_{2} .
$$

Where $W_{2}$ represents the output interference rejection coefficient.

For such an object with uncertainty, the design steps of QFT are as follows:

1) Selecting characteristic frequency points, object template and nominal object.

2) Plotting QFT performance index boundary curve.

3) Shaping and design of controller.

A Simple Design Method of QFT Controller. In the traditional quantitative feedback theory, the controller design is a repeated trial process. It requires designers with design experience. It is relatively difficult for beginners. To avoid this problem, this paper designs a simple method for QFT controller design.

Observing Eq. (2)-(4) can be found that they can be converted into the form of sensitivity function $|S(j \omega)|=|1 /(1+C(j \omega) P(j \omega))|$ and form of complement sensitivity function $|T(j \omega)|=C(j \omega) P(j \omega) /(1+C(j \omega) P(j \omega)) \mid$

After the conversion, at each characteristic frequency point:

$$
\left\{\begin{array}{l}
\left|S\left(j \omega_{i}\right)\right| \leq \min \left\{W_{1 x}, W_{2}\right\} \\
\left|T\left(j \omega_{i}\right)\right| \leq M_{0}
\end{array} .\right.
$$

At each characteristic frequency point, the object and the controller can be represented as complex exponential form: $P\left(j \omega_{i}\right)=p\left(\omega_{i}\right) e^{j \theta\left(\omega_{i}\right)}, C\left(j \omega_{i}\right)=c\left(\omega_{i}\right) e^{j \phi\left(\omega_{i}\right)}$, then Eq. (5) can be transformed into:

$$
\left\{\begin{array}{l}
c^{2}\left(\omega_{i}\right) p^{2}\left(\omega_{i}\right)+2 c\left(\omega_{i}\right)\left[p\left(\omega_{i}\right) \cos (\phi+\theta)\right]+\left(1-1 / \min ^{2}\left\{W_{1 x}, W_{2}\right\}\right) \geq 0 . \\
c^{2}\left(\omega_{i}\right)\left[p^{2}\left(\omega_{i}\right)\left(1-1 / M_{0}^{2}\right)\right]+2 c\left(\omega_{i}\right)\left[p\left(\omega_{i}\right) \cos (\phi+\theta)\right]+1 \geq 0
\end{array} .\right.
$$

To meet the performance indexes, we can make the two equations in Eq. (6) equal:

$$
c\left(\omega_{i}\right)=\frac{1}{p\left(\omega_{i}\right)} \frac{M_{0}}{\min \left\{W_{1 x}, W_{2}\right\}} .
$$


Eq. (7) gives the controller amplitude that satisfies multiple performance indexes. Next, we can choose a specific controller structure, using least squares curve fitting method to obtain the controller parameters.

\section{Implicit Generalized Predictive Self - Tuning Control Algorithm and Improvement}

Implicit generalized predictive contro ${ }^{[10]}$ does not need to identify the model parameters, but directly to identify the parameters of the control law according to the data of input and output.

In generalized predictive control, the CARIMA model is generally as follows ${ }^{[1]}$ :

$$
A\left(z^{-1}\right) y(k)=z^{-d} B\left(z^{-1}\right) u(k)+C\left(z^{-1}\right) \xi(k) / \Delta \text {. }
$$

Where $y(k), u(k)$ and $\xi(k)$ represent the output, input and white noise at k step, and d represents the delay.

The system output at $k+j$ step is:

$$
y(k+j)=G_{j}\left(z^{-1}\right) \Delta u(k+j-1)+f(k+j) .
$$

Where $f(k+j)$ represents the output response based on the data of past input and output.

The reference trajectory is calculated as follows:

$$
\left\{\begin{array}{l}
y_{r}(k)=y(k) \\
y_{r}(k+j)=\alpha^{j} y(k)+\left(1-\alpha^{j}\right) w
\end{array} .\right.
$$

Where $\alpha \in[0,1)$ represents the output softening factor and $w$ represents the reference.

The objective function used in [12] is:

$$
J=E\left\{\left[G \Delta U+f-Y_{r}\right]^{T}\left[G \Delta U+f-Y_{r}\right]+\lambda \Delta U^{T} \Delta U\right\} .
$$

When the traditional GPC algorithm is used in the superheated steam temperature control system, the output will appear varying degree of overshoot. Also, the amplitude of the control is constrained in this system.

In this paper, the function of the control is added to Eq. (11):

$$
J=E\left\{\left[G \Delta U+f-Y_{r}\right]^{T}\left[G \Delta U+f-Y_{r}\right]+\lambda \Delta U^{T} \Delta U+\beta U^{T} U\right\} .
$$

This is done for suppressing the amplitude of the control and the excessive overshoot. Where $\beta$ is the coefficient of input suppression.

$$
\begin{aligned}
& U=M+N \Delta U . M=\left[\begin{array}{lll}
u(k-1) & \cdots & u(k-1)
\end{array}\right]_{1 \times N_{u}}^{T} . \\
& N=\left[\begin{array}{cccc}
1 & 0 & \cdots & 0 \\
1 & 1 & \cdots & 0 \\
\vdots & \vdots & 1 & 0 \\
1 & 1 & \cdots & 1
\end{array}\right]_{N_{u} \times N_{u}}
\end{aligned}
$$

Where $N_{u}$ is the step of control.

\section{IGPC-QFT Cascade Control Algorithm}

For the characteristics of the superheated steam temperature control system, many scholars used the generalized predictive control algorithm to control the system ${ }^{[13]}$. The generalized predictive control can solve the problem of changed model parameters. But when the delay of the controlled object is large and the model parameters change greatly with the load, the output will appear varying degree of overshoot. In the superheated steam temperature control system, the delay and inertia of the leading region are much smaller than the whole system. And interference and uncertainty are usually included in the leading region. Therefore, the IGPC-QFT cascade control algorithm is proposed. The controller 
designed by QFT is used to reduce influence of uncertainty and reject the interference in the leading region, so that the leading steam temperature can be controlled in advance. The improved IGPC controller is used in the inert region to overcome the large delay and inertia of the system, restrict overshoot and make the main steam temperature track reference. IGPC has a well tracking performance, so there is no need to design F filter in QFT.

Based on the above ideas, an IGPC-QFT cascade control algorithm is designed. The structure is shown in Fig. 2.

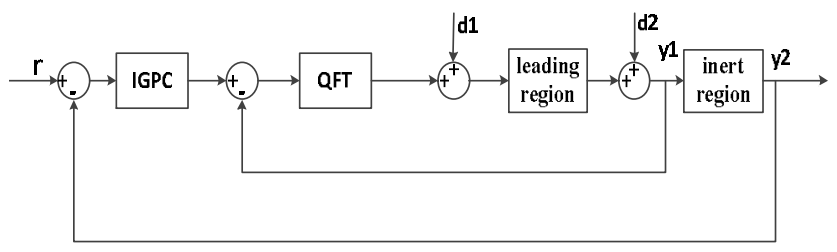

Fig. 2 Structure of IGPC-QFT cascade control

Where $\mathrm{r}$ represents the reference of the main steam temperature, $d_{1}, d_{2}$ represent the interference, $y_{1}$ represents the leading steam temperature, $y_{2}$ represents the main steam temperature.

\section{Simulation Study}

Four typical models ${ }^{[14]}$ are given in Table 1.

\begin{tabular}{ccc}
\multicolumn{2}{c}{ Table 1 Model parameters under different load } \\
\hline load & leading region & $\begin{array}{c}\text { inert } \\
\text { region }\end{array}$ \\
\hline $37 \%$ & $-\frac{5.072}{(1+28 s)^{2}}$ & $\frac{1.048}{(1+56.6 s)^{8}}$ \\
\hline $50 \%$ & $-\frac{3.067}{(1+25 s)^{2}}$ & $\frac{1.119}{(1+42.1 s)^{7}}$ \\
\hline $75 \%$ & $-\frac{1.657}{(1+20 s)^{2}}$ & $\frac{1.202}{(1+27.1 s)^{7}}$ \\
\hline $100 \%$ & $-\frac{0.815}{(1+18 s)^{2}}$ & $\frac{1.276}{(1+18.4 s)^{6}}$ \\
\hline
\end{tabular}

In QFT, controller design mainly according to the uncertain model of leading region. Here, the uncertain model of the leading region can be expressed as:

$$
\vartheta=\left\{P(s)=-\frac{k}{(a s+1)^{2}}: k \in[0.815,5.072], a \in[18,28]\right\} .
$$

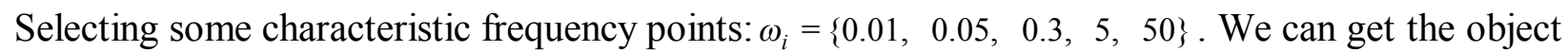
template. Selecting a model in extreme case as nominal object :

$$
P_{0}(s)=-\frac{0.815}{(1+18 s)^{2}} .
$$

Setting robust stability margin index as:

$$
\left|\frac{C(j \omega) P(j \omega)}{1+C(j \omega) P(j \omega)}\right| \leq 1.2 \text {. }
$$

Setting the input interference rejection index as:

$$
\left|\frac{P(j \omega)}{1+C(j \omega) P(j \omega)}\right| \leq 0.03 .
$$

Setting the input interference rejection index as: 


$$
\left|\frac{1}{1+C(j \omega) P(j \omega)}\right| \leq 0.03 \text {. }
$$

According to the above set of performance indexes, we can plot the corresponding performance index boundary, and then take the intersection to form a composite boundary. Composite boundary and not shaped open loop frequency response curve are shown in Fig. 3.

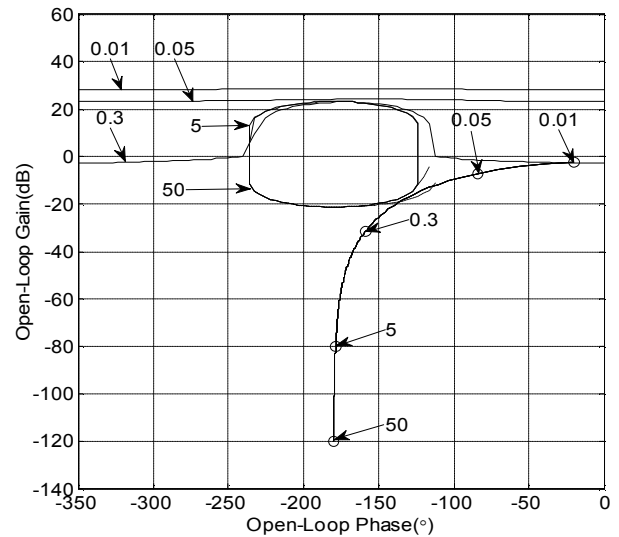

Fig. 3 Composite boundary and not shaped open-loop frequency response

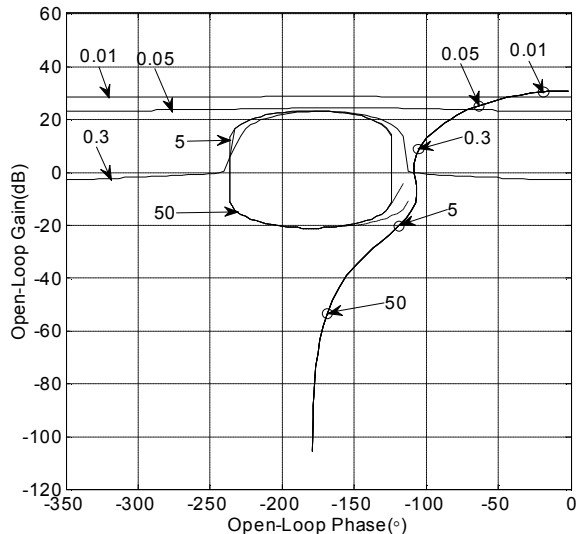

Fig. 4 Composite boundary and shaped open-loop frequency response

In order to meet the requirement of corresponding performance index boundary, we can use Eq. (7) to obtain the amplitude of satisfactory controller at the characteristic frequency point:

$c\left(\omega_{i}\right)=[43.06,45.26,48.13,368.32,807.43]$.

Then, selecting a specific structure of controller. Least squares curve fitting method is used to identify the controller parameters. We can get the satisfactory controller:

$$
C(s)=\frac{43.34(1.9 s+1)}{(0.1 s+1)} \text {. }
$$

Then, the composite boundary curve and the shaped open-loop frequency response curve are plotted, as shown in Fig. 4. It can be seen from Fig. 4, the controller designed by the simple method can meet the requirement of the composite boundary curve.

In the simulation, we set the desired output of the system as a square wave which varies in the range of $[1,2]$. The load of the system is gradually increased from $37 \%$ to $100 \%$. The parameters of IGPC: The step of prediction is $\mathrm{N}=24$, the step of control is $N_{u}=2$, the weighting coefficient of control is $\lambda=0.1$, the softening factor of output is $\alpha=0.8$, the coefficient of input suppression is $\beta=0.3$. The initial value of recursive least squares method: $g_{j-1}=1, P=10^{5} \mathrm{I}$, forgetting factor is $\lambda_{0}=1$.

By MATLAB simulation, comparing with traditional GPC-P algorithm (proportional controller $K_{p}=20$ ), the simulation results are shown in Fig. 5 and Fig. 6.
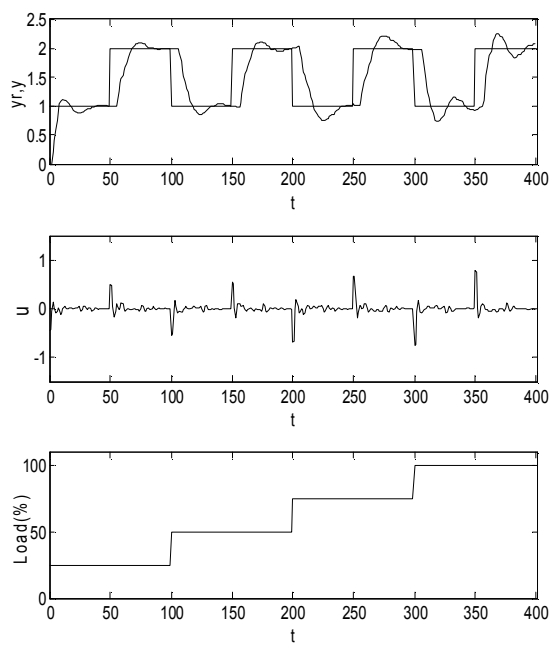
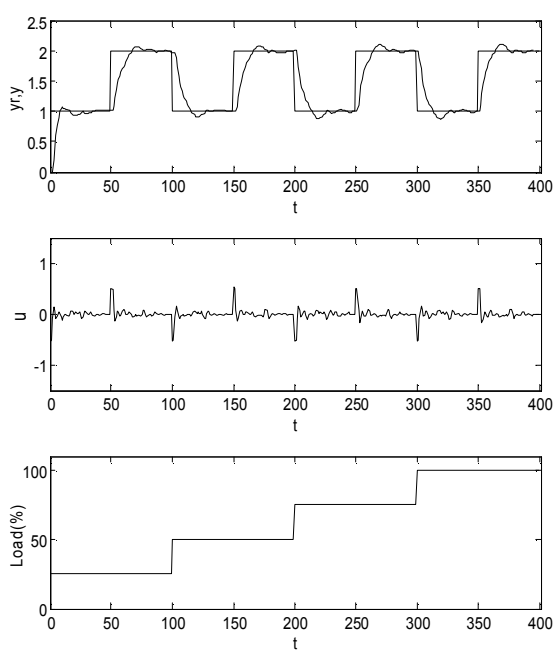
Fig.5 GPC-P cascade control

Fig.6 IGPC-QFT cascade control

It can be seen from the comparison that, the output of the system under the traditional GPC-P control algorithm has varying degree of overshoot. The time to reach $5 \%$ steady state is long. The IGPC-QFT cascade control algorithm makes the overshoot small. The time to reach $5 \%$ steady state is short. When the parameters of the model change with the load, the system can maintain well dynamic performance.

\section{Conclusions}

For the unique characteristics of superheated steam temperature control system in the thermal power plant, the IGPC-QFT cascade control strategy proposed in this paper overcomes the large time-delay, large inertia and time-varying property of the system, restricts the overshoot and obtains a good tracing performance. Meanwhile, this paper also proposes a simple design for QFT controller, which avoids the complex shaping process. It can be known from the simulation results that this control strategy can be well applied in the superheated steam temperature control system.

\section{Acknowledgements}

This work was financially supported by National Natural Science Foundation (NNSF) of China (51405349).

\section{References}

[1] Sreenivasulu G, Reddy S N: submitted to Iete Journal of Research (2015).

[2] Benxian Xiao, et al: submitted to International Journal of Information Technology \& Computer Science (2014).

[3] Pérez R R, Geddes A, Clegg A: submitted to International Journal of Adaptive Control \& Signal Processing (2012).

[4] Li Deng, Yan Huang, Minrui Fei, et al: submitted to Chinese Journal of Scientific, Instrument (2014). In Chinese.

[5] Horowitz, Isaac: submitted to International Journal of Robust \& Nonlinear Control (2001).

[6] Woodbury T, Valasek J: AIAA Guidance, Navigation, and Control Conference (2015), p. 1-21.

[7] Yan Wang, Yufu Qin, Shengrong Guo: submitted to China Mechanical Engineering (2013). In Chinese.

[8] Purohit H, Goldsztejn A, Jermann C, et al: submitted to International Journal of Robust \& Nonlinear Control (2016).

[9] Shuangxin Wang, Fei He, Rujiu Liu, et al: submitted to Electric Machines and Control (2016). In Chinese.

[10]Guoyong Li, Lijuan Yang: Neural fuzzy predictive control and its MATLAB implementation. Publishing House of Electronics Industry (2013). In Chinese.

[11]Clarke D W, Mohtadi C, Tuffs P S: submitted to Automatica (1987).

[12] G B Liu: submitted to Journal of Heilongjiang Institute of Technology (2012).

[13]Linxiao Geng, Xiaoguo Jing: submitted to Thermal Power Generation (2015).In Chinese.

[14]Junjie Gu, Jianqiang Li, Daming Gao, et al: Thermal control system. China Electric Power Press, (2011). In Chinese. 